\title{
Paricalcitol versus
}

Calcitriol + Cinacalcet for the

Treatment of Secondary

Hyperparathyroidism in Chronic

Kidney Disease in China: A Cost-Effectiveness Analysis

\author{
Zhuolin Zhang ${ }^{1}$, Lele Cai ${ }^{1}$, Hong Wu ${ }^{1,2}$, Xinglu X ${ }^{1}$, Wenqing Fang ${ }^{3}$, Xuan $\mathrm{He}^{3}$, \\ Xiao $\mathrm{Wang}^{3}$ and $\mathrm{Xin} \mathrm{Li}^{1,3,4 *}$
}

OPEN ACCESS

Edited by:

Kevin Lu,

University of South Carolina,

United States

Reviewed by:

Ivana Stasevic Karlicic,

Clinic for Mental Disorders "Dr Laza

Lazarevic", Serbia

Guojun Sun,

Zhejiang University of

Technology, China

Jianzhou Yan,

China Pharmaceutical

University, China

*Correspondence:

Xin $\mathrm{Li}$

xinli@njmu.edu.cn

Specialty section:

This article was submitted to

Health Economics,

a section of the journal

Frontiers in Public Health

Received: 19 May 2021

Accepted: 25 June 2021

Published: 21 July 2021

Citation:

Zhang Z, Cai L, Wu H, Xu X, Fang W,

He $X$, Wang $X$ and Li $X$ (2021)

Paricalcitol versus

Calcitriol + Cinacalcet for the

Treatment of Secondary

Hyperparathyroidism in Chronic

Kidney Disease in China: A

Cost-Effectiveness Analysis.

Front. Public Health 9:712027.

doi: 10.3389/fpubh.2021.712027
${ }^{1}$ Department of Clinical Pharmacy, School of Pharmacy, Nanjing Medical University, Nanjing, China, ${ }^{2}$ Department of Pharmacy, Lianyungang Second People's Hospital, Lianyungang, China, ${ }^{3}$ Department of Health Policy, School of Health Policy and Management, Nanjing Medical University, Nanjing, China, ${ }^{4}$ Center for Global Health, School of Public Health, Nanjing Medical University, Nanjing, China

Background: Chronic Kidney Disease (CKD) is a global chronic disease with increasing prevalence in recent years, particularly CKD accompanied by Secondary Hyperparathyroidism (SHPT) leads to reduced quality of life, increased mortality, a considerable economic burden for patients and society. The aim of this study was to investigate the cost-effectiveness analysis of paricalcitol vs. calcitriol + cinacalcet for CKD patients with SHPT in China in 2020.

Methods: A Markov model was conducted employing data derived from published literature, clinical trials, official sources, and tertiary public hospital data in China, based on a 10-year horizon from the perspective of the healthcare system. Calcitriol + Cinacalcet was used as the reference group. CKD stage 5 (CKD-5) dialysis patients suffering from SHPT were included in the study. Effectiveness was measured in quality-adjusted life years (QALYs). The discount rate (5\%) was applied to costs and effectiveness. Sensitivity analysis was performed to confirm the robustness of the findings.

Results: The base case analysis demonstrated that Patients treated with paricalcitol could gain an increase in utility (0.183 QALYs) and require fewer expenditures (6925.612 yuan). One-way sensitivity analysis was performed to showed that impact factors were the price of cinacalcet, the hospitalization costs of patients with paricalcitol and calcitriol, the costs and utilities of hemodialysis and the costs of calcitriol, the costs of paricalcitol regardless of period. Probabilistic simulation analysis displayed when willingness-to-pay was $¥ 217113$, the probability that Paricalcitol was dominant is $96.20 \%$.

Conclusion: The results showed that paricalcitol administrated to treat patients diagnosed with Secondary hyperparathyroidism in Chronic Kidney Disease, compared to calcitriol and cinacalcet, might be dominant in China.

Keywords: paricalcitol, calcitriol, cinacalcet, secondary hyperparathyroidism, chronic kidney disease, costeffectiveness analysis 


\section{BACKGROUND}

Chronic Kidney Disease (CKD) is a global chronic disease with gradually renal function loss. The number of individuals diagnosed with CKD and who die from CKD in China ranks top worldwide (1). The prevalence of CKD, even exceeding that of diabetes mellitus (2), has increased progressively from 10.8 to $11.6 \%$ during $2012-2018$ in China $(3,4)$. The aging population deteriorates the prevalence of CKD in China, as well as a physiological decline in renal function and rising prevalence of Hypertension and Diabetes (3,5). A cross-sectional survey conducted in China revealed that $\sim 3$ million CKD patients arrived in the end-stage renal disease (ESRD) stage over 3 years (4), where CKD patients are necessary to receive renal replacement treatment (RRT) (6). A large population of potential patients exists in China (4), as estimated, the prevalence of ESRD grown at a rate of $1.95 \%$ from 2015 to 2020 in Nanjing (7), a medium-sized city of China, imposing enormous pressure on the health care system due to productivity loss and premature death $(8,9)$.

Secondary hyperparathyroidism (SHPT) is a common serious complication in dialysis patients (10), over $70 \%$ advanced CKD patients are diagnosed with SHPT (11). As reported, about $60 \%$ of maintaining hemodialysis patients were affected by different levels of severity of SHPT, where the standard-reaching rate was about merely 55\% (12). Due to renal dysfunction resulting in increased endogenous calcitriol and serum calcium, while decreased serum phosphorus (13), SHPT is characterized by increased serum parathyroid hormone (PTH) and parathyroid hyperplasia (14), risk factors of cardiovascular disease and fracture, resulting in lower quality of life, and higher mortality $(10,15,16)$.

In 2017, total Medicare expenditure for CKD and ESRD in the US was high, approaching over 120 billion dollars (17). The same situation occurs in China in that the medical spending of CKD patients was 27.646 billion RMB in 2016, accounted for $6.50 \%$ of total health spending. For dialysis patients, the medical expenditure approached 911 million RMB, 75.6\% of that was covered by urban basic health insurance. The economic burden of ESRD rises over time (9). It has been established that the medical expenditures of ESRD are multiplying at a mean annual rate of $5.8 \%$ in Nanjing (7). Treatment of CKD and ESRD is a heavy burden for patients, for family, and the health care system. Undoubtedly, Complications like SHPT, CVD exacerbate the economic burden for dialysis patients as well (18-20).

Based on the guideline-recommended by Kidney Disease: Improving Global Outcomes (KDIGO), Vitamin D Receptor Activators (VDRA) and calcimimetics are the optimal strategies of SHPT treatment, complemented to decrease PTH, prevent hypocalcemia, improve survival, and reduce cardiovascular disease $(15,21)$. Approximately $60 \%$ of patients undergoing hemodialysis and peritoneal dialysis were treated with Calcitriol, a non-selective Vitamin D (18). However, the adverse effects of Calcitriol due to non-selective mechanisms, hyperphosphatemia, and hypercalcemia causing vascular and soft tissue calcification (13) - the risk factors of mortality, restricts the effectiveness of calcitriol on the treatment of $\operatorname{SHPT}(10,22,23)$. Cinacalcet, the first and the only calcimimetics launched in China, approving for the treatment of maintaining dialysis (12). It has been documented that Cinacalcet has the advantage of quick onset in declining serum calcium, serum phosphorus, and $\mathrm{PTH}$, meanwhile, reduce volume and size of parathyroid glands, hardly causing hyperphosphatemia and hypercalcemia $(15,24)$. Cinacalcet was commonly adopted when Vitamin D was poorly controlled (12).

Paricalcitol, a synthetic analog of vitamin D, less hypercalcemia and hypercalcemia, effectively decreases PTH, particularly administrated intravenously compared to the Calcitriol $(15,25,26)$. As reported, in the long term, paricalcitol has a significant survival advantage and decreases hospitalization (4, 27-29). Notably, paricalcitol could be effective for calcitriol-resistant hemodialysis patients $(23,30)$.

Therefore, here we choose calcitriol and cinacalcet as references to explore the cost-effectiveness analysis of Paricalcitol. The cost-effectiveness of paricalcitol has been proved in Italian, US, UK, Brazil, German (13, 31-34). We aim to explore the cost-effectiveness of paricalcitol in China.

\section{METHODS}

\section{Model Design}

A Markov model (Figure 1) was conducted by Microsoft ${ }^{\circledR}$ Excel (Excel 2019 version) based on the model Nujiten (31) published. We invited local experts to confirm the Markov model is reasonable by requiring nephrology and pharmacoeconomics specialists' advice. The Markov model was selected for the following reasons: Markov model allows us to assess the costeffectiveness of paricalcitol in the long term by defining a series of health states and health state transitions between these states to simulate disease process and combining consumable costs with health outcomes in every health states (35), moreover, it could take complications into considerations (31).

We assumed that a hypothetical cohort of 10,000 patients step into the model to calculate the standard error to make probabilistic sensitivity analysis and set that each cycle of the model was 1 year. All patients were in the "peritoneal dialysis" state (PD) or "hemodialysis" (HD) state at the beginning, the proportion distribution was extracted from the annual report on kidney in China (18). Patients could transfer to "Transplantation"

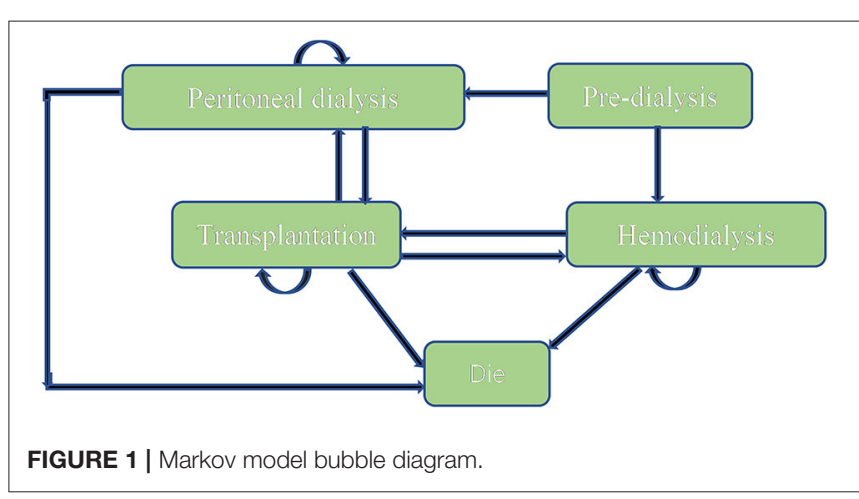


state or "Dead" state, also could stay the original state after 1-year cycle. "Transplantation" and "peritoneal dialysis," "Transplantation," and "hemodialysis" were possible to convert each other. Importantly, "Dead" was an absorbed state and could not move back to other states. We hypothesized that the time horizon of 10 years since a study conducted in China $(n=$ 153) reported that the survival rates from 5 years to 10 years for hemodialysis and peritoneal hemodialysis elderly patients dropped to 18.4 and $2.6 \%$ (36), which was appropriate because it covered the entire process from the moment they were diagnosed. The annual discount rate of costs and health outcomes was $5 \%$.

\section{Cost Assessment}

As shown in Table 1, the medical costs incorporated in this model included the costs of management of SHPT (paricalcitol, calcitriol, and cinacalcet), the costs of inpatient due to common complications (cardiovascular disease, fracture, and a series of complications), and the costs of management of ESRD (peritoneal dialysis, hemodialysis, transplantation) from the perspective of the health care system. The total cost per one patient was assessed once a year. All costs were presented and measured as Renminbi (RMB) in 2020.

\section{Costs of Management of SHPT}

The cost and dosage for each drug were obtained from official sources and published literature. Calcitriol was given twice daily, each dose is $0.25 \mathrm{ug}$. The dose of cinacalcet is $44.7 \mathrm{mg}$ once daily (38). The daily dose of paricalcitol could not be stable until after 18 weeks based on the literature (25). Paricalcitol was administrated by three times a week before meeting the standard, after that altering to once weekly (37). The cost of each drug recorded in detail were summarized in Table 2.

\section{Costs of Hospitalization}

The costs of hospitalization of CKD-5 patients were from a tertiary public hospital, located in Jiangsu Province in China during 2019-2020. Jiangsu province is located on the southeast coast of China. The eastern region like Jiangsu ranks top in the medical resources density index and healthcare services utilization in China in 2016, therefore, it is a good representative.

TABLE 1 | Inputs to model for base case analysis and sensitivity analysis.

\begin{tabular}{|c|c|c|c|c|c|}
\hline \multirow[t]{2}{*}{ Costs (yuan/year) } & \multirow[t]{2}{*}{ Base case analysis } & \multicolumn{2}{|c|}{ One-way sensitivity analysis } & \multirow[t]{2}{*}{ Distribution } & \multirow[t]{2}{*}{ References } \\
\hline & & $\min$ & $\max$ & & \\
\hline \multicolumn{6}{|l|}{ Drug costs } \\
\hline Paricalcitol 1st year & 21766.86 & 10448.09 & 26120.23 & Gamma $(\alpha=10000.00, \beta=2.18)$ & $(25,37)$ \\
\hline Paricalcitol beyond 1st year & 18562.86 & 8910.17 & 22275.43 & Gamma $(\alpha=10000.00, \beta=1.86)$ & $(37)$ \\
\hline Calcitriol & 3942.00 & 1892.16 & 4730.40 & Gamma $(\alpha=10000.00, \beta=0.39)$ & Official sources \\
\hline Cinacalcet & 27740.00 & 13315.20 & 33288.00 & Gamma $(\alpha=10000.00, \beta=2.77)$ & (38) \\
\hline \multicolumn{6}{|l|}{ Renal replacement costs } \\
\hline $\begin{array}{l}\text { Kidney transplantation } 1 \text { st } \\
\text { year }\end{array}$ & 188697.90 & 150958.32 & 226437.48 & Gamma $(\alpha=560.06, \beta=336.92)$ & Hospital data \\
\hline $\begin{array}{l}\text { Kidney transplantation } \\
\text { beyond } 1 \text { st year }\end{array}$ & 99228.25 & 47629.56 & 119073.89 & Gamma $(\alpha=194.63, \beta=509.83)$ & Hospital data \\
\hline HD & 67200.00 & 32256.00 & 80640.00 & Gamma $(\alpha=10000.00, \beta=6.72)$ & (39) \\
\hline PD & 51600.00 & 24768.00 & 61920.00 & Gamma $(\alpha=10000.00, \beta=5.16)$ & (39) \\
\hline \multicolumn{6}{|l|}{ Inpatient costs } \\
\hline Calcitriol + Cinacalcet & 19375.57 & 15500.46 & 23250.68 & Gamma $(\alpha=58.26, \beta=332.59)$ & Hospital data \\
\hline Paricalcitol & 19557.85 & 15646.28 & 23469.42 & Gamma $(\alpha=10.90, \beta=1793.48)$ & Hospital data \\
\hline \multicolumn{6}{|l|}{ Utility (QALY/year) } \\
\hline HD & 0.60 & 0.54 & 0.66 & Beta $(\alpha=1638.584, \beta=1092.39)$ & $(40)$ \\
\hline PD & 0.60 & 0.54 & 0.66 & Beta $(\alpha=1638.584, \beta=1092.39)$ & $(40)$ \\
\hline Kidney transplantation & 0.84 & 0.76 & 0.92 & Beta $(\alpha=7238.162, \beta=1378.70)$ & $(32)$ \\
\hline Dead & 0 & - & - & - & - \\
\hline Discount rate & $5 \%$ & $0 \%$ & $8 \%$ & - & - \\
\hline Risk adjustment & 0.23 & & & & $(32)$ \\
\hline
\end{tabular}

TABLE 2 | The costs of SHPT management including paricalcitol, calcitriol, and cinacalcet.

\begin{tabular}{|c|c|c|c|c|}
\hline Medications & Cost/tablet ( $¥)$ & Weekly cost ( $¥)$ & Annual cost before up-to-standard ( $¥$ ) & Annual cost after up-to-standard $(¥)$ \\
\hline Paricalcitol & 178 & 534 & 21766.86 & 18562.86 \\
\hline Calcitriol & 5.4 & 75.6 & 3942 & 3942 \\
\hline Cinacalcet & 38 & 532 & 27740 & 27740 \\
\hline
\end{tabular}


The reason why we choose a single tertiary public hospital is that the few hospitals administrate expensive paricalcitol and cinacalcet, we used the data to show Chinese practical clinical setting by cost. The inclusion criteria of CKD-5D patients are as follows: (1) CKD-5D patients diagnosed with SHPT; (2) Eighteen years of age and above; (3) CKD-5D patients are accompanied by other common complications; The exclusion criteria are as follows: (1) Cross-use of paricalcitol, calcitriol, and cinacalcet during the hospital stay. (2) The patients with other diseases have no association with SHPT, such as depression and tumors.

The annual number of hospitalizations was derived from a US-setting study (28). The average annual number of hospitalizations of paricalcitol, calcitriol was 2.40, 2.61, respectively. Here, we assume that Cinacalcet was consistent with calcitriol, which was a conservative approach toward cinacalcet to perform an analysis in this study due to unavailable data.

\section{Costs of Management of ESRD}

The costs of transplantation of CKD-5 patients were from a tertiary public hospital, located in Jiangsu Province in China during 2019-2020. The direct medical cost involved kidney source fees, bed charges, nursing care fees, operating expenses, laboratory fees, inspection fees, drug fees, and other fees (material fees) in the first year of receiving kidney transplantation. The medical costs during next year focused on following-up visit costs, including drug fees, laboratory fees, inspection fees, and other fees (material fees).

\section{Transition Probabilities Including Mortalities}

The majority of transition probabilities between health state was from an Italian study (31), partly corrected by the local proportion of HD and PD patients in China (18). In details, the annual probabilities change from kidney transplantation to dialysis was $5.00 \%$ (41), as official data in China reported (18), $91.46 \%$ of kidney transportation would choose hemodialysis, others would receive peritoneal dialysis. Therefore, we can calculate that $4.60 \%$ of kidney transplantation patients receive hemodialysis and $0.40 \%$ of that choose peritoneal dialysis. The transition probabilities were shown in Tables 3, 4 .

The mortalities data were from same literature (31), widely used in the different clinical settings (31-33), that the mortality

TABLE 3 | Transition probabilities between different states in patients with calcitriol and cinacalcet.

\begin{tabular}{lcccc}
\hline & HD & PD & Transplantation & Die \\
\hline 1 year & & & & \\
HD & 0.829 & 0 & 0.033 & 0.138 \\
PD & 0 & 0.867 & 0.033 & 0.100 \\
Transplantation & 0.0460 & 0.00403 & 0.920 & 0.030 \\
$>$ 1 year & & & & \\
HD & 0.829 & 0 & 0.033 & 0.138 \\
PD & 0 & 0.817 & 0.033 & 0.150 \\
Transplantation & 0.0460 & 0.00403 & 0.934 & 0.016
\end{tabular}

rate of paricalcitol decreased by $15 \%$ (27), compared to those treated with calcitriol. This historical cohort study enrolling a large number patients revealed that paricalcitol have greater survival advantage over calcitriol.

\section{Utility}

The health utility scores of ESRD patients from the Singapore cross-sectional survey (40) were adopted where the Chinese population accounts for over half the proportion. However, we judged that the health utility score of kidney transplantation population from the UK setting (32) could be applied to the Chinese kidney transplantation population due to unavailable data. Those are measured based on EQ-5D. Health outputs were expressed in terms of quality-adjusted life years (QALYs), which can assess intervention strategy in terms of life time and life quality to reflect the health state of subjects better.

\section{Base Case Analysis}

Base case analysis results were expressed as incremental cost-effectiveness ratio (ICER), which was calculated as incremental costs/incremental QALYs. Based on the World Health Organization (WHO) recommendations, if the ICER $<$ per capita Pross Domestic Product (GDP), the treatment strategy is totally cost effective; if the per capita GDP < ICER $<$ three times per capita GDP, the therapy strategy is acceptable in terms of economic evaluation; if the ICER > three times per capita GDP, we can consider that the therapy strategy is not cost effective. In this study, we assumed that the paricalcitol regimen was cost-effective when the ICER was less than three times per capita gross domestic product (GDP). According to the latest data from the 2020 National Economic and Social Development Statistical Bulletins, the per capita GDP was 7.2447 yuan (42).

TABLE 4 | Transition probabilities between different states in patients with paricalcitol.

\begin{tabular}{lcccc}
\hline & HD & PD & Transplantation & Die \\
\hline 1 year & & & & \\
HD & 0.8576 & 0 & 0.0254 & 0.117 \\
PD & 0 & 0.8896 & 0.0254 & 0.085 \\
Transplantation & 0.0354 & 0.0031 & 0.9355 & 0.026 \\
$>$ 1 year & & & & \\
HD & 0.8576 & 0 & 0.0254 & 0.117 \\
PD & 0 & 0.8466 & 0.0254 & 0.128 \\
Transplantation & 0.0354 & 0.0031 & 0.9475 & 0.014 \\
\hline
\end{tabular}

TABLE 5 | Expected costs and QALYs of two treatment strategies, incremental costs, and incremental QALYs between two treatment strategies, ICERs.

\begin{tabular}{lcccc}
\hline & $\begin{array}{c}\text { Costs } \\
\text { (Yuan) }\end{array}$ & $\begin{array}{c}\text { Utility } \\
\text { (QALYs) }\end{array}$ & $\boldsymbol{\Delta C}$ & $\boldsymbol{\Delta U}$ \\
\hline Paricalcitol & 472596.007 & 2.855 & -6925.612 & 0.183 \\
Calcitriol + Cinacalcet & 479521.619 & 2.672 & &
\end{tabular}




\section{Sensitivity Analysis}

The one-way sensitivity analysis and probabilistic simulation analysis were conducted to ensure the robustness of the results. One-way sensitivity analysis was performed by changing a parameter every time to highest or lowest values. A Tornado graph was used to shows the influential variables-the results of one-way sensitivity. Due to unavailable $95 \%$ confidence interval (CI), the inpatient expenditures were set to fluctuate between -20 and $+20 \%$, the range of utility started from -10 to $+10 \%$.
Of note, we assume that the price of medicines and devices (including transplantation costs and dialysis costs) involved in disease progression exhibited a mean decrease of $52 \%$ because the centralized tender system of medicines and medical devices is being established in China (43).

Probabilistic simulation analysis was conducted by varying all parameters within set different distributions simultaneously in 1000 Monte Carlo simulation iterations to illustrate the results of uncertain analysis and build a cost-effectiveness acceptability

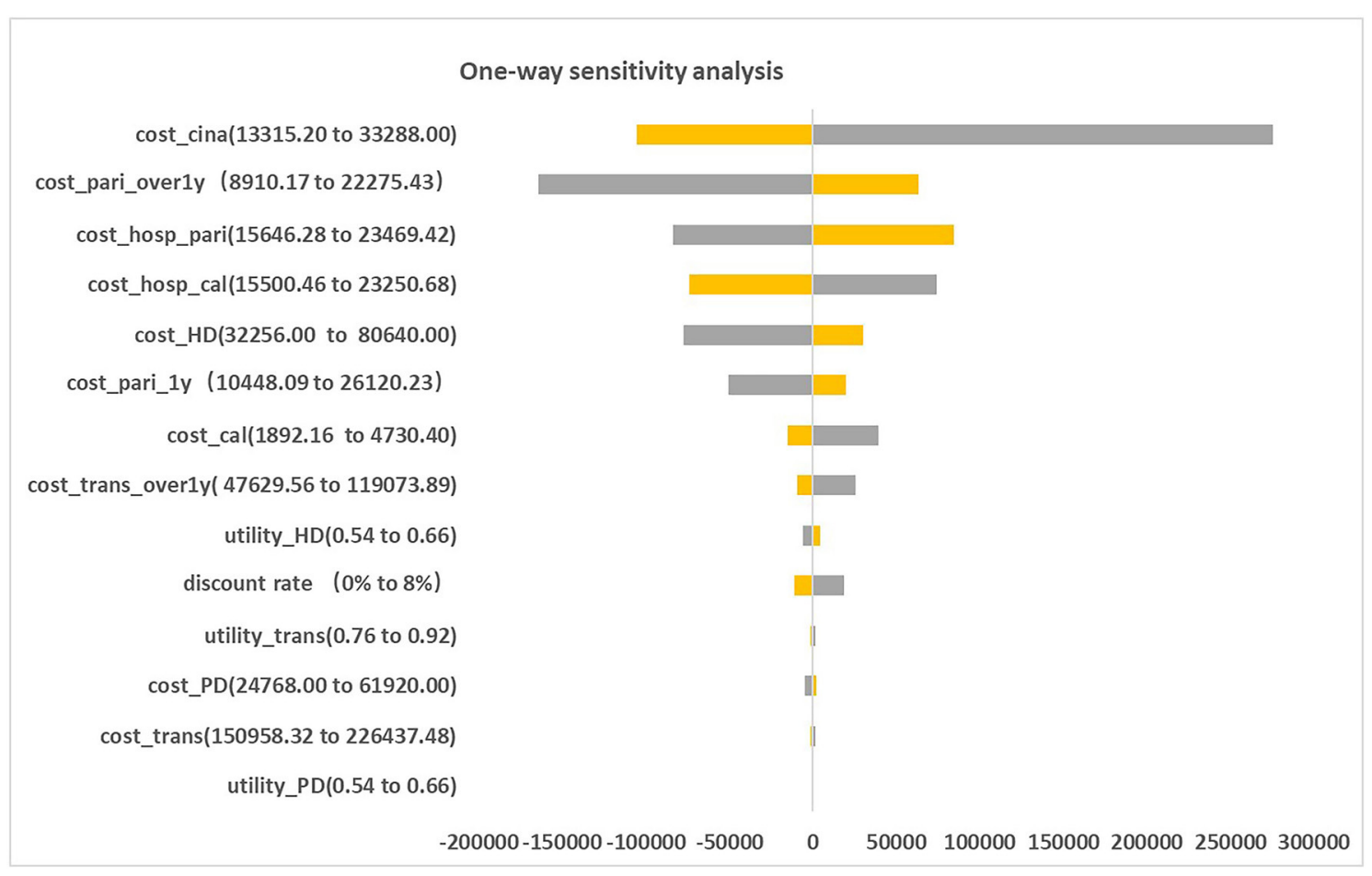

FIGURE 2 | Tornado diagram for two therapies: paricalcitol vs. calcitriol and cinacalcet.



FIGURE 3 | The cost-effectiveness plane illustrated ICERs between paricalcitol and calcitriol + cinacalcet in 1,000 iterations of Monte Carlo simulation. 
curve. In this study, we set costs, utility followed gamma distribution $(31,33)$, beta distribution, respectively. The value of willingness-to-pay was defined as three times GDP per capita, which was 21.7113 yuan. Here we report that EVPI (expected value of perfect information), interpreted as the average of maximum net monetary benefits, estimates the expected values of uncertainty by combining perfect information with the current known information. We still assume that the benefit patients cohort is 10,000 patients.

\section{RESULTS}

\section{Base Case Analysis}

The results of the base case analysis were summarized in Table 5. Total costs of CKD-5D patients accompanied with SHPT, treated with paricalcitol (472596.007 yuan) paid lower than those with calcitriol and cinacalcet (479521.619 yuan), total utilities as well (2.855 QALYs vs. 2.672 QALYs), in 10 years in the Chinese Healthcare System. Compared to regimen Calcitriol and Cinacalcet, patients who choose Paricalcitol as a regimen need to pay $<6925.612$ yuan, but gain an increase in Utility $(0.183$ QALYs). Paricalcitol was dominant as compared to calcitriol and cinacalcet.

\section{Sensitivity Analysis}

Figure 2 presented the tornado diagram of one-way sensitivity analysis showing the ICERs in descending order were sensitive to which parameters. In this present study, parameters that might affect ICER. The results showed that this model was relatively robust. The most impactful factor on the total outcome was the price of cinacalcet. The results of probabilistic simulation analysis and were displayed in Figures 3, 4. When willingnessto-pay was 217113 yuan, $96.20 \%$ of patients were willing to pay for paricalcitol. Figure 5 displayed the EVPI altering at a variety of willingness-to-pay thresholds.

\section{DISCUSSION}

This is the first study that revealing the cost-effectiveness of paricalcitol in China healthcare system settings. The findings of base case analysis showed that paricalcitol was preferred for CKD-5D patients accompanied with SHPT in China compared to the combination use of calcitriol and cinacalcet, with ICER was negative. This study shows that CKD-5 individuals with SHPT administrated by paricalcitol are an optimal strategy for CKD patients with SHPT in China. The one-way sensitivity analysis suggested that the price of cinacalcet was the most influential factor, the probabilistic sensitivity analysis showed that the probability of paricalcitol was dominant is $96.20 \%$ when the threshold of willingness-to-pay was 217113 yuan. Our findings are in line with the previous results done in CKD patients with SHPT from other national studies.

On the other hand, the one-way sensitivity analysis results revealed our model was relatively robust. The main reason that led to this phenomenon was that the magnitudes of decrease in

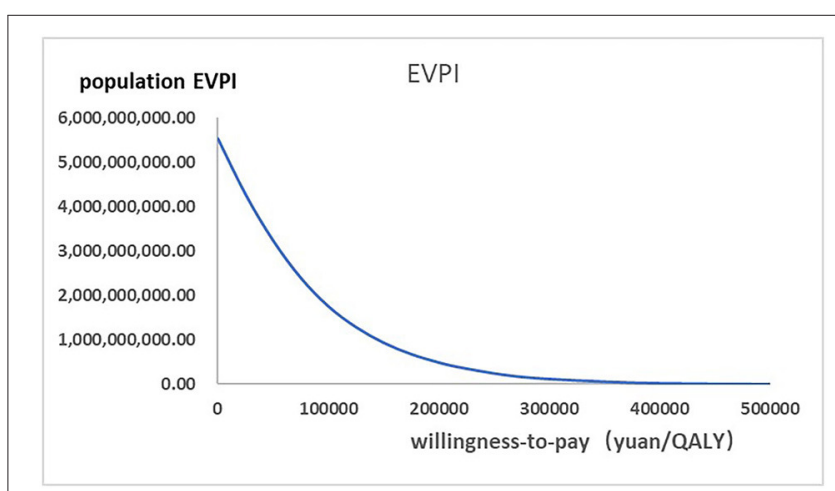

FIGURE 5 | The expected values combining current information and perfect information were shown in the EVPI graph at a variety of willingness-to-pay thresholds.

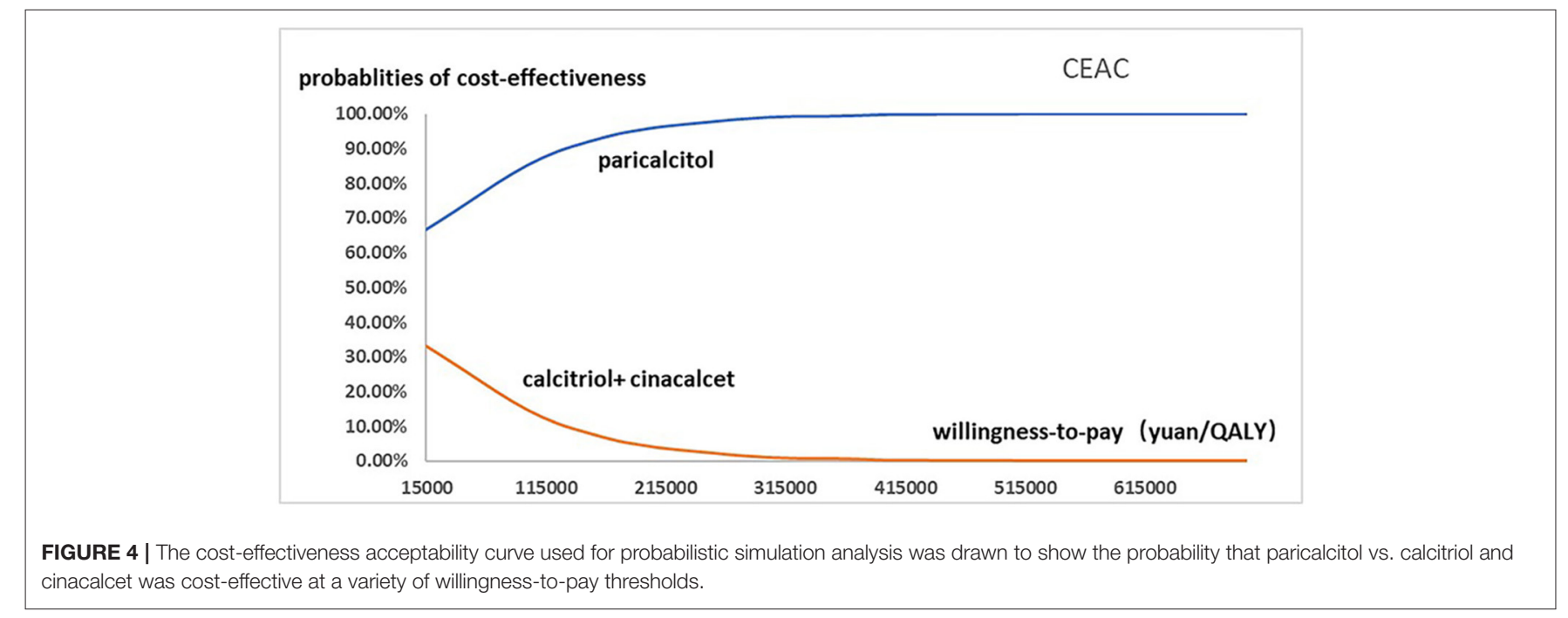


costs of cinacalcet in the model were large (52\%). Furthermore, we found it particularly depended on the dosage of paricalcitol after achieving the therapeutic range.

It has been reported that paricalcitol could extend survival and decrease the number of hospitalizations $(26,28)$. Other advantages have been gradually recognized. For renal transplant recipients, a clinical trial reveals that paricalcitol could decrease proteinuria, which could reduce the risk of kidney transplantation failure (44). Paricalcitol currently is approved to be used in adults, while clinical trials had proven to be effective for children regardless of oral and intravenous $(45,46)$, besides this, some studies showed that paricalcitol could potentially treat cardiac dysfunction and diabetic nephropathy $(47,48)$, which suggests the scope of the applicable population should be extended. Furthermore, patients preferred to receive the injection of paricalcitol in the process of getting dialysis due to convenience (11), compared to taking oral calcitriol every day, which can reduce the probability of forgetting to take drugs and increase medication adherence to better control SHPT (49).

With an increase in time of dialysis, vitamin D therapy may be ineffective, parathyroidectomy (PTX) could be considered (50), contributing to improving quality of life (51) and reducing all-cause mortality in ESRD patients with SHPT (52). There are few studies to discuss the cost-effectiveness of PTX, usually compared with cinacalcet $(53,54)$, which provided new ideas that the cost-effectiveness of PTX for refractory secondary hyperparathyroidism could be investigated in China. Additionally, here we try to discuss more on cinacalcet and other calcimimetics. Cinacalcet has significantly apparent treatment efficacy for severe SHPT patients, it can be used to decrease PTH before receiving PTX and it is a substitute PTX treatment strategy as well (55). Notably, cinacalcet reduces the risk of undergoing PTX (19), also could obtain favorable outcomes for patients with recurrent SHPT after receiving parathyroidectomy (56). Therefore, cinacalcet, a potential therapeutic agent, should be further assessed. A randomized clinical trial (57) demonstrates a novel calcimimetics called etelcalcetide is not inferior to cinacalcet for moderate and severe SHPT patients, in addition, etelcalcetide is administrated intravenous injection to improve adherence and reduce the probability of gastrointestinal adverse effects. However, etelcalcetide has not been launched in China, which is expected to improve cost-effectiveness of medication therapy soon.

However, there are existing problems in terms of treating SHPT in China. Firstly, only $20 \%$ of ESRD patients received RRT in China, especially concentrated in large and medium-sized cities (58), which indicated that there are significant geographic differences in ESRD treatment, besides the large population base of CKD patients with SHPT, which may lead to accelerated conditions due to the lack of timely and adequate treatment. Moreover, ESRD patients with complications might achieve worse outcomes, like productivity loss, the absence of caregivers, and even premature death (9). Based on China Kidney Disease Network 2016 annual data report (18), patients with CKD, particularly with SHPT, are associated with increased risk of morbidity and mortality in cardiovascular disease (49), which causes higher medical costs compared to other complications. However, high healthcare costs can not contribute to ideal therapeutic efficacy. According to this study, governments and the relevant departments should pay close attention to optimize medical resource allocation, to reduce the burden of patients. Besides that, over $80 \%$ of ESRD patients choose HD as renal replacement treatment in Asia, which parallel to the global conditions, to achieve maximum outcomes of ESRD patients (59). However, HD has worsened outcomes and higher costs than renal transplantation (59), which prompted us to conduct a cost-effectiveness analysis between $\mathrm{HD}$ and renal transplantation to provide optimal clinical decisions, which may update awareness and prescribing habits of doctors without considering kidney donations.

This study has several limitations. First, we did some assumptions far away from the real world. Especially, the dosage of drugs, health utility scores of kidney transplantation, and transition probabilities in different health states were not derived from Chinese populations. We could not obtain available data due to no similar studies had been conducted in China. For example, a study revealed that the 1-year survival rate was higher, while the 5-year survival rate was lower than that we adopted (60), whereas we could not confirm that specific clinical setting of the study so that we could not support that. However, according to the requirements of the International Conference on Coordination for Human Drugs (ICH), paricalcitol was approved by National Medical Products Administration (NMPA) in China without local clinical trials, which could demonstrate that the efficacy and safety of paricalcitol in Chinese populations was as same as those in other countries. Therefore, it is reasonable that we adopted the data from published literature from Italia. Second, merely paricalcitol, calcitriol, and cinacalcet were included in the analysis, a possible future of improvement could also incorporate Erythropoietin-treated anemia (a common complication of CKD) and phosphate binders into the analysis. We should be aware of the importance of phosphate control which is significant to mortality and treatment outcomes, moreover, calcium-containing phosphate binders were no longer recommended by China guideline (12) to prevent vascular and soft tissue calcification. Third, we accounted for direct costs using a healthcare perspective, we did not include indirect costs (like productivity loss due to ESRD patients are usually all unproductive, it will be offset from the perspective of the healthcare system and transportation costs) and other costs such as the costs of treating adverse effects. The reason why we did not take adverse effects costs into account is that the adverse effect of paricalcitol, calcitriol and cinacalcet are usually managed by adjusting dosages, which needs real-world data to calculate, we have sufficient data to calculate, which need further exploration.

\section{CONCLUSION}

The results demonstrated that paricalcitol used for the treatment of secondary hyperparathyroidism in Chronic Kidney Disease when compared to calcitriol and cinacalcet, 
would be cost-effective from the perspective of the healthcare system in China over 10 years.

\section{DATA AVAILABILITY STATEMENT}

The original contributions presented in the study are included in the article/supplementary material, further inquiries can be directed to the corresponding author.

\section{REFERENCES}

1. GBD Chronic Kidney Disease Collaboration. Global, regional, and national burden of chronic kidney disease, 1990-2017: a systematic analysis for the Global Burden of Disease Study 2017. Lancet. (2020) 395:709-33. doi: 10.1016/S0140-6736(20)30045-3

2. Hill NR, Fatoba ST, Oke JL, Hirst JA, O'Callaghan CA, Lasserson DS, et al. Global prevalence of chronic kidney disease - a systematic review and metaanalysis. PLoS ONE. (2016) 11:e0158765. doi: 10.1371/journal.pone.0158765

3. Wang F, He K, Wang J, Zhao MH, Li Y, Zhang L, et al. Prevalence and risk factors for CKD: a comparison between the adult populations in China and the United States. Kidney Int Rep. (2018) 3:1135-43. doi: 10.1016/j.ekir.2018.05.011

4. Zhang L, Wang F, Wang L, Wang W, Liu B, Liu J, et al. Prevalence of chronic kidney disease in China: a cross-sectional survey. Lancet. (2012) 379:815-22. doi: 10.1016/S0140-6736(12)60033-6

5. Gansevoort RT, Correa-Rotter R, Hemmelgarn BR, Jafar TH, Heerspink HJL, Mann JF, et al. Chronic kidney disease and cardiovascular risk: epidemiology, mechanisms, and prevention. Lancet. (2013) 382:339-52. doi: 10.1016/S0140-6736(13)60595-4

6. Zhang L, Zuo L. Current burden of end-stage kidney disease and its future trend in China. Clin Nephrol. (2016) 86(Suppl 1):27-8. doi: 10.5414/CNP86S104

7. Sun L, Zou LX, Han CY, Huang HM, Tan Z, Gao M, et al. Forecast of the incidence, prevalence and burden of end-stage renal disease in Nanjing, China to the Year 2025. BMC Nephrol. (2016) 17:60. doi: 10.1186/s12882-0160269-8

8. Lv J, Zhang L. Prevalence and disease burden of chronic kidney disease. $A d v$ Exp Med Biol. (2019) 1165:3-15. doi: 10.1007/978-981-13-8871-2_1

9. Wang V, Vilme H, Maciejewski ML, Boulware LE. The economic burden of chronic kidney disease and end-stage renal disease. Semin Nephrol. (2016) 36:319-30. doi: 10.1016/j.semnephrol.2016.05.008

10. Zhang $\mathrm{T}, \mathrm{Ju} \mathrm{H}$, Chen $\mathrm{H}$, Wen W. Comparison of paricalcitol and calcitriol in dialysis patients with secondary hyperparathyroidism: a metaanalysis of randomized controlled studies. Ther Apher Dial. (2019) 23:73-9. doi: 10.1111/1744-9987.12760

11. Hauber B, Caloyeras J, Posner J, Brommage D, Belozeroff V, Cooper K. Hemodialysis patients' preferences for the management of secondary hyperparathyroidism. BMC Nephrol. (2017) 18:254. doi: 10.1186/s12882-017-0665-8

12. National Clinical Research Center of Kidney Diseases. Experts consensus on calcimimetics in patients with chronic kidney disease. Chin J Nephrol. (2018) 34:703-8. doi: 10.1016/j.diabres.2017.02.032

13. Rosery H, Bergemann R, Marx SE, Boehnke A, Melnick J, Sterz R, et al. Health-economic comparison of paricalcitol, calcitriol and alfacalcidol for the treatment of secondary hyperparathyroidism during haemodialysis. Clin Drug Investig. (2006) 26:629-38. doi: 10.2165/00044011-200626110-00002

14. Cozzolino M, Tomlinson J, Walsh L, Bellasi A. Emerging drugs for secondary hyperparathyroidism. Exp Opin Emerg Drugs. (2015) 20:197-208. doi: $10.1517 / 14728214.2015 .1018177$

15. Mizobuchi M, Ogata H, Koiwa F. Secondary hyperparathyroidism: pathogenesis and latest treatment. Ther Apher Dial. (2019) 23:309-318. doi: 10.1111/1744-9987.12772

16. El-Kholey MMAE, Ibrahim GES, Elshahat OI, El-Kannishy G. Impact of subtotal parathyroidectomy on clinical parameters and quality of life

\section{AUTHOR CONTRIBUTIONS}

ZZ, LC, and XL designed the whole study. ZZ, HW, LC, and $\mathrm{XX}$ were responsible for collecting and analyzing data. ZZ, LC, $\mathrm{WF}, \mathrm{XW}$, and $\mathrm{XH}$ contributed to preparing the original draft. $\mathrm{ZZ}, \mathrm{XX}$, and $\mathrm{XL}$ took the responsibility of review and editing. All authors have read and agreed to the published version of the manuscript.

in hemodialysis patients with secondary hyperparathyroidism. Endocrinol Metab. (2019) 34:367-73. doi: 10.3803/EnM.2019.34.4.367

17. Saran R, Robinson B, Abbott KC, Bragg-Gresham J, Chen X, Gipson D, et al. US renal data system 2019 annual data report: epidemiology of kidney disease in the United States. Am J Kidney Dis. (2020) 75(1 Suppl 1):A6-7. doi: 10.1053/j.ajkd.2019.09.003

18. Zhang L, Zhao M, Zuo L, Wang Y, Yu F, Zhang H, et al. China kidney disease network (CK-NET) 2016 annual data report. Kidney Int Suppl. (2011) 10:e97-185. doi: 10.1016/j.kisu.2020.09.001

19. Belozeroff V, Chertow GM, Graham CN, Dehmel B, Parfrey PS, Briggs AH. Economic evaluation of cinacalcet in the United States: the EVOLVE trial. Value Health. (2015) 18:1079-87. doi: 10.1016/j.jval.2015.08.007

20. Kalantar-Zadeh K, Hollenbeak CS, Arguello R, Snyder S, Ashfaq A. The cost- effectiveness of extended-release calcifediol versus paricalcitol for the treatment of secondary hyperparathyroidism in stage 3-4 CKD. J Med Econ. (2020) 23:308-15. doi: 10.1080/13696998.2019.1693385

21. Jean G, Souberbielle JC, Chazot C. Vitamin D in chronic kidney disease and dialysis patients. Nutrients. (2017) 9:328. doi: 10.3390/nu9040328

22. Ong LM, Narayanan P, Goh HK, Manocha AB, Ghazali A, Omar M, et al. Randomized controlled trial to compare the efficacy and safety of oral paricalcitol with oral calcitriol in dialysis patients with secondary hyperparathyroidism. Nephrology. (2013) 18:194-200. doi: 10.1111/nep.12029

23. Llach F, Yudd M. Paricalcitol in dialysis patients with calcitriol-resistant secondary hyperparathyroidism. Am J Kidney Dis. (2001) 38(5 Suppl 5):S4550. doi: 10.1053/ajkd.2001.28114

24. Komaba H, Nakanishi S, Fujimori A, Tanaka M, Shin J, Shibuya K, et al. Cinacalcet effectively reduces parathyroid hormone secretion and gland volume regardless of pretreatment gland size in patients with secondary hyperparathyroidism. Clin J Am Soc Nephrol. (2010) 5:2305-14. doi: 10.2215/CJN.02110310

25. Sprague SM, Llach F, Amdahl M, Taccetta C, Batlle D. Paricalcitol versus calcitriol in the treatment of secondary hyperparathyroidism. Kidney Int. (2003) 63:1483-90. doi: 10.1046/j.1523-1755.2003.00878.x

26. Akizawa T, Akiba T, Hirakata H, Kinugasa E, Tominaga $Y$, Fukagawa M, et al. Comparison of paricalcitol with maxacalcitol injection in Japanese hemodialysis patients with secondary hyperparathyroidism. Ther Apher Dial. (2015) 19:225-34. doi: 10.1111/1744-9987.12242

27. Teng M, Wolf M, Lowrie E, Ofsthun N, Lazarus JM, Thadhani R. Survival of patients undergoing hemodialysis with paricalcitol or calcitriol therapy. $N$ Engl J Med. (2003) 349:446-56. doi: 10.1056/NEJMoa022536

28. Dobrez DG, Mathes A, Amdahl M, Marx SE, Melnick JZ, Sprague SM. Paricalcitol-treated patients experience improved hospitalization outcomes compared with calcitriol-treated patients in real-world clinical settings. Nephrol Dial Transplant. (2004) 19:1174-81. doi: 10.1093/ndt/gfh123

29. Zheng Z, Shi H, Jia J, Li D, Lin S. Vitamin D supplementation and mortality risk in chronic kidney disease: a meta-analysis of 20 observational studies. BMC Nephrol. (2013) 14:199. doi: 10.1186/1471-2369-14-199

30. Tonbul HZ, Solak Y, Atalay H, Turkmen K, Altintepe L. Efficacy and tolerability of intravenous paricalcitol in calcitriol-resistant hemodialysis patients with secondary hyperparathyroidism: 12-month prospective study. Ren Fail. (2012) 34:297-303. doi: 10.3109/0886022X.2011.647298

31. Nuijten M, Roggeri DP, Roggeri A, Novelli P, Marshall TS. Health economic evaluation of paricalcitol $(\mathbb{R})$ versus cinacalcet + calcitriol (oral) in Italy. [corrected]. Clin Drug Investig. (2015) 35:229-38. doi: $10.1007 /$ s40261-014-0264-4 
32. Nuijten M, Andress DL, Marx SE, Curry AS, Sterz R. Cost Effectiveness of paricalcitol versus a non-selective vitamin $\mathrm{D}$ receptor activator for secondary hyperparathyroidism in the UK: a chronic kidney disease markov model. Clin Drug Investig. (2010) 30:545-57. doi: 10.2165/11536310-000000000-00000

33. Nuijten M, Andress DL, Marx SE, Sterz R. Chronic kidney disease Markov model comparing paricalcitol to calcitriol for secondary hyperparathyroidism: a US perspective. Curr Med Res Opin. (2009) 25:1221-34. doi: 10.1185/03007990902844097

34. Menezes FG, Abreu RM, Itria A. Cost-effectiveness analysis of paricalcitol versus calcitriol for the treatment of SHPT in dialytic patients from the SUS perspective. J Bras Nefrol. (2016) 38:313-9. doi: 10.5935/0101-2800.20160048

35. Liu GG. China Guidelines for Pharmacoeconomic Evaluations. Beijing: China Market Press (2020).

36. Chen A, Zhao B, Xu L, Sun Y, Wang S, Li T, et al. Analysis of causes of death in elderly patients undergoing hemodialysis and peritoneal dialysis. Chin J Geriatr. (2020) 39:1050-4. doi: 10.3760/cma.j.issn.0254-9026.2020. 09.014

37. Staniforth ME, Cheng SC, Coyne DW. Once-weekly intravenous paricalcitol in the treatment of secondary hyperparathyroidism in hemodialysis patients. Clin Nephrol. (2005) 63:454-60. doi: 10.5414/CNP63454

38. Sharma A, Ketteler M, Marshall TS, Khan SS, Schumock GT. Comparative cost analysis of management of secondary hyperparathyroidism with paricalcitol or cinacalcet with low-dose vitamin D in hemodialysis patients. J Med Econ. (2013) 16:1129-36. doi: 10.3111/13696998.2013. 823092

39. Wang W, Liang H, Lu W. Analysis on treatment burden of end-stage renal disease patients and related policy suggestions. Chinese Health Resources. (2018) 21:121-6. doi: 10.13688/j.cnki.chr.2018.17794

40. Yang F, Griva K, Lau T, Vathsala A, Lee E, Ng HJ, et al. Health-related quality of life of Asian patients with end-stage renal disease (ESRD) in Singapore. Qual Life Res. (2015) 24:2163-71. doi: 10.1007/s11136-015-0964-0

41. Javier V, Maria J, Rosa R, Jose I M, Angel LMF, Jose L, et al. Returning to hemodialysis after kidney allograft failure: a survival study with propensity score matching. Nephrol Dial Transplant. (2018) 34:667-72. doi: $10.1093 / \mathrm{ndt} / \mathrm{gfy} 215$

42. National Bureau of Statistics of China. Statistical Communique of the People's Republic of China on the 2020 National Economic and Social Development. Statistical Communique of the People's Republic of China on the 2020 National Economic and Social Development (stats.gov.cn) (2021). (accessed May 17, 2021).

43. CHINA NEW. Drug Prices of Centralized Drug Procurement Fell by an Average of 52\% in Pilot Cities. (2019). Available online at: https://m.chinanews.com/ wap/detail/sp/sp/shipin/cns/2019/11/27/news9019083.shtml (accessed April 14, 2021).

44. Oblak M, Mlinšek G, Kandus A, Buturović-Ponikvar J, Arnol M. Paricalcitol versus placebo for reduction of proteinuria in kidney transplant recipients: a double-blind, randomized controlled trial. Transpl Int. (2018) 31:1391-404. doi: $10.1111 /$ tri.13323

45. Webb NJA, Lerner G, Warady BA, Dell KM, Greenbaum LA, Ariceta G, et al. Efficacy and safety of paricalcitol in children with stages 3 to 5 chronic kidney disease. Pediatr Nephrol. (2017) 32:1221-32. doi: 10.1007/s00467-017-3579-6

46. Greenbaum LA, Benador N, Goldstein SL, Paredes A, Melnick JZ, Mattingly S, et al. Intravenous paricalcitol for treatment of secondary hyperparathyroidism in children on hemodialysis. Am J Kidney Dis. (2007) 49:814-23. doi: 10.1053/j.ajkd.2007.03.008

47. Tamayo M, Martín-Nunes L, Val-Blasco A, GM-Piedras MJG, Navarro-García JA, Lage E, et al. Beneficial effects of paricalcitol on cardiac dysfunction and remodelling in a model of established heart failure. Br J Pharmacol. (2020) 177:3273-90. doi: 10.1111/bph.15048

48. Nakhoul N, Thawko T, Farber E, Dahan I, Tadmor H, Nakhoul R, et al. The therapeutic effect of active vitamin $\mathrm{D}$ supplementation in preventing the progression of diabetic nephropathy in a diabetic mouse model. J Diabetes Res. (2020) 2020:7907605. doi: 10.1155/2020/7907605

49. Alfieri C, Regalia A, Zanoni F, Vettoretti S, Cozzolino M, Messa P. The importance of adherence in the treatment of secondary hyperparathyroidism. Blood Purif. (2019) 47:37-44. doi: 10.1159/000492918

50. Kakani E, Sloan D, Sawaya BP, El-Husseini A, Malluche HH, Rao M. Longterm outcomes and management considerations after parathyroidectomy in the dialysis patient. Semin Dial. (2019) 32:541-52. doi: 10.1111/sdi.12833

51. Plas WY, Dulfer RR, Engelsman AF, Vogt L, Borst MH, Ginhoven TM, et al. Effect of parathyroidectomy and cinacalcet on quality of life in patients with end-stage renal disease-related hyperparathyroidism: a systematic review. Nephrol Dial Transplant. (2017) 32:1902-8. doi: 10.1093/ndt/gfx044

52. Apetrii M, Goldsmith D, Nistor I, Siriopol D, Voroneanu L, Scripcariu D, et al. Impact of surgical parathyroidectomy on chronic kidney disease-mineral and bone disorder (CKD-MBD) - a systematic review and meta-analysis. PLoS ONE. (2017) 12:e0187025. doi: 10.1371/journal.pone.0187025

53. Komaba H, Moriwaki K, Goto S, Yamada S, Taniguchi M, Kakuta T, et al. Costeffectiveness of cinacalcet hydrochloride for hemodialysis patients with severe secondary hyperparathyroidism in Japan. Am J Kidney Dis. (2012) 60:262-71. doi: 10.1053/j.ajkd.2011.12.034

54. Narayan R, Perkins RM, Berbano EP, Yuan CM, Neff RT, Sawyers ES, et al. Parathyroidectomy versus cinacalcet hydrochloride-based medical therapy in the management of hyperparathyroidism in ESRD: a cost utility analysis. Am J Kidney Dis. (2007) 49:801-13. doi: 10.1053/j.ajkd.2007.03.009

55. Susantitaphong P, Vadcharavivad S, Susomboon T, Singhan W, Dumrongpisutikul N, Jakchairoongruang $\mathrm{K}$, et al. The effectiveness of cinacalcet: a randomized, open label study in chronic hemodialysis patients with severe secondary hyperparathyroidism. Ren Fail. (2019) 41:326-33. doi: 10.1080/0886022X.2018.1562356

56. Zitt E, Rix M, Torres PU, Fouque D, Jacobson SH, Pétavy F, et al. Effectiveness of cinacalcet in patients with recurrent/persistent secondary hyperparathyroidism following parathyroidectomy: results of the ECHO study. Nephrol Dial Transplant. (2011) 26:1956-61. doi: 10.1093/ndt/ gfq641

57. Block GA, Bushinsky DA, Cheng S, Cunningham J, Dehmel B, Drueke TB, et al. Effect of etelcalcetide vs cinacalcet on serum parathyroid hormone in patients receiving hemodialysis with secondary hyperparathyroidism: a randomized clinical trial. JAMA. (2017) 317:156-64. doi: $10.1001 /$ jama.2016.19468

58. Wu H, Li Q, Cai Y, Zhang J, Cui W, Zhou Z. Economic burden and cost-utility analysis of three renal replacement therapies in ESRD patients from Yunnan Province, China. Int Urol Nephrol. (2020) 52:573-9. doi: 10.1007/s11255-020-02394-1

59. Liew A. Perspectives in renal replacement therapy: haemodialysis. Nephrology. (2018) 23(Suppl 4):95-9. doi: 10.1111/nep.13449

60. Sun Y, Wang Y, Yu W, Zhuo Y, Yuan Q, Wu X. Association of dose and frequency on the survival of patients on maintenance of hemodialysis in China: a Kaplan-Meier and cox-proportional hazard model analysis. Med Sci Monit. (2018) 24:5329-37. doi: 10.12659/MSM.909404

Conflict of Interest: The authors declare that the research was conducted in the absence of any commercial or financial relationships that could be construed as a potential conflict of interest.

Copyright (C) 2021 Zhang, Cai, Wu, Xu, Fang, He, Wang and Li. This is an openaccess article distributed under the terms of the Creative Commons Attribution License (CC BY). The use, distribution or reproduction in other forums is permitted, provided the original author(s) and the copyright owner(s) are credited and that the original publication in this journal is cited, in accordance with accepted academic practice. No use, distribution or reproduction is permitted which does not comply with these terms. 